\title{
SLICING AND INTERSECTION THEORY FOR CHAINS MODULO $v$ ASSOCIATED WITH REAL ANALYTIC VARIETIES
}

BY

\author{
ROBERT M. HARDT( $\left.{ }^{1}\right)$
}

\begin{abstract}
In a real analytic manifold a $k$ dimensional (real) analytic chain is a locally finite sum of integral multiples of chains given by integration over certain $k$ dimensional analytic submanifolds (or strata) of some $k$ dimensional real analytic variety. In this paper, for any integer $\nu \geq 2$, the concepts and results of [6] on the continuity of slicing and the intersection theory for analytic chains are fully generalized to the modulo $\nu$ congruence classes of such chains.
\end{abstract}

1. Introduction. For a separable real analytic manifold $M$, a real analytic mapping $f: M \rightarrow \mathbf{R}^{n}$, and a $k$ dimensional analytic chain $T$ in $M[4,4.2 .28]$ with $k \geq n$, it was show $\mathrm{n}$ in $[6,4.3]$ that the slice function $\langle T, f, \cdot\rangle$ is continuous on the set of points $y$ in $\mathbf{R}^{n}$ for which

$$
\operatorname{dim}\left(f^{-1}\{y\} \cap \operatorname{spt} T\right) \leq k-n \text { and } \operatorname{dim}\left(f^{-1}\{y\} \cap \operatorname{spt} \partial T\right) \leq k-n-1 .
$$

Geometrically, for almost all $y$ in $\mathbf{R}^{n}$, the slice $\langle T, f, y\rangle$ is the $k-n$ dimensional analytic chain in $M$ given by oriented integration, counting multiplicities, along the fiber $f^{-1}\{y\} \cap$ spt $T$.

For any integer $\nu \geq 2$, two $k$ dimensional analytic chains $T_{1}$ and $T_{2}$ are congruent modulo $\nu$ if there exists a third analytic chain $Q$ such that $T_{1}-T_{2}$ $=\nu Q$. The resulting congruence classes are called $k$ dimensional analytic chains modulo $\nu$ in $M$. With such a congruence class $S$ we associate the set $\operatorname{spt}^{\nu} S=$ $\bigcap_{T \in S}$ spt $T$. Generalizing $[6,4.3]$, we prove in 4.1 our main result:

Slicing Modulo $\nu$ Theorem. Suppose $S$ is a $k$ dimensional analytic chain modulo $\nu$ in $M, f$ is an analytic map of $M$ into $\mathbf{R}^{n}, k \geq n$, and

Received by the editors March 23, 1972.

AMS (MOS) subject classifications (1970). Primary 28A75, 32B20, 32C05; Secondary $14 \mathrm{~A} 10,32 \mathrm{C} 30$.

Key words and phrases. Analytic chain (modulo $\nu$ ), slice (modulo $\nu$ ), support (modulo $\nu$ ), dimension, intersection theory, flat chain (modulo $\nu$ ), rectifiable current, mass.

(1) This work was supported in part by National Science Foundation grants GP16807 and GP29321. 
$\mathbf{R}^{n} \cap\left\{y: \operatorname{dim}\left(f^{-1}\{y\} \cap \mathrm{spt}^{\nu} S\right) \leq k-n\right.$ and $\left.\operatorname{dim}\left(f^{-1}\{y\} \cap \mathrm{spt}^{\nu} \partial S\right) \leq k-n-1\right\}$. Then there exists a continuous mapping from $Y$ into the $k-n$ dimensional analytic chains modulo $\nu$ in $M$ sucb that for every $y \in Y$ the value $\langle S, f, y\rangle^{\nu}$, which we call the slice modulo $\nu$ of $S$ in $f^{-1}\{y\}$, satisfies the condition:

If $T$ is a $k$ dimensional analytic chain in $M$ belonging to $S$ with $\operatorname{dim}\left(f^{-1}\{y\} \cap \operatorname{spt} T\right) \leq k-n$ and $\operatorname{dim}\left(f^{-1}\{y\} \cap \operatorname{spt} \partial T\right) \leq k-n-1$, then $\langle T, f, y\rangle$ belongs to $\langle S, f, y\rangle^{\nu}$.

As an elementary example consider the four linear maps $g_{1}, g_{2}, g_{3}: \mathbf{R} \rightarrow \mathbf{R}^{2}$, $f: \mathbf{R}^{2} \rightarrow \mathbf{R}$ defined by $g_{1}(y)=(y, 0), g_{2}(y)=\left(-y, 3^{1 / 2} y\right), g_{3}(y)=\left(-y,-3^{1 / 2} y\right)$ for $y \in \mathbf{R}$ and $f(y, z)=y$ for $(y, z) \in \mathbf{R}^{2}$, the oriented half-line $H=\mathbf{E}^{1} L\{y: y>0\}$ in $\mathbf{R}$, and the one dimensional analytic chain

$$
T=g_{1 \#} H+g_{2 \#} H+g_{3 \#} H
$$

in $\mathbf{R}^{2}$ (see Figure 1). We compute $\partial T=-3 \delta_{(0,0)}$ and

$$
\begin{aligned}
& \langle T, f, a\rangle=-\delta_{\left(a,-3^{1 / 2} a\right)}-\delta_{\left(a, 3^{1 / 2} a\right)} \text { for } a<0, \\
& \langle T, f, b\rangle=\delta_{(b, 0)} \text { for } b>0 \quad \text { (see Figure 2). }
\end{aligned}
$$

Hence $\langle T, f,$.$\rangle is continuous on \mathbf{R} \sim\{0\}=\mathbf{R} \sim f($ spt $\partial T)$, in accord with $[6,4.3]$; however,

$$
\lim _{y \rightarrow 0_{-}}\langle T, f, y\rangle=-2 \delta_{(0,0)} \neq \delta_{(0,0)}=\lim _{y \rightarrow 0^{+}}\langle T, f, y\rangle .
$$

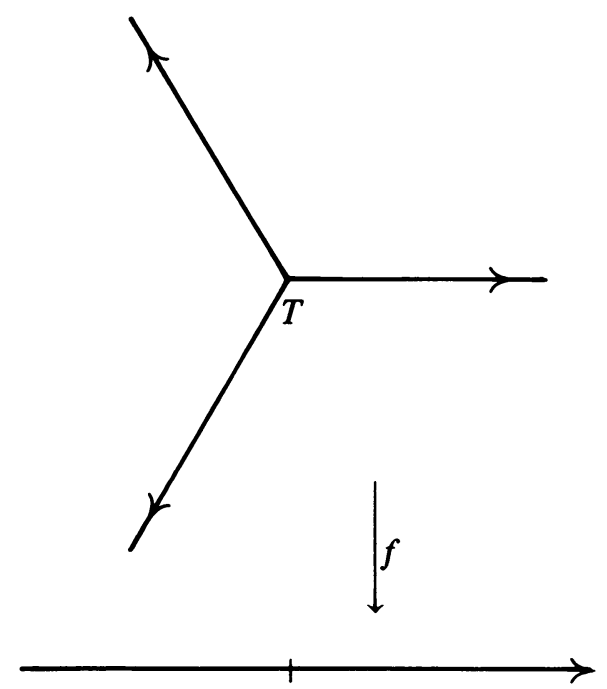

Figure 1

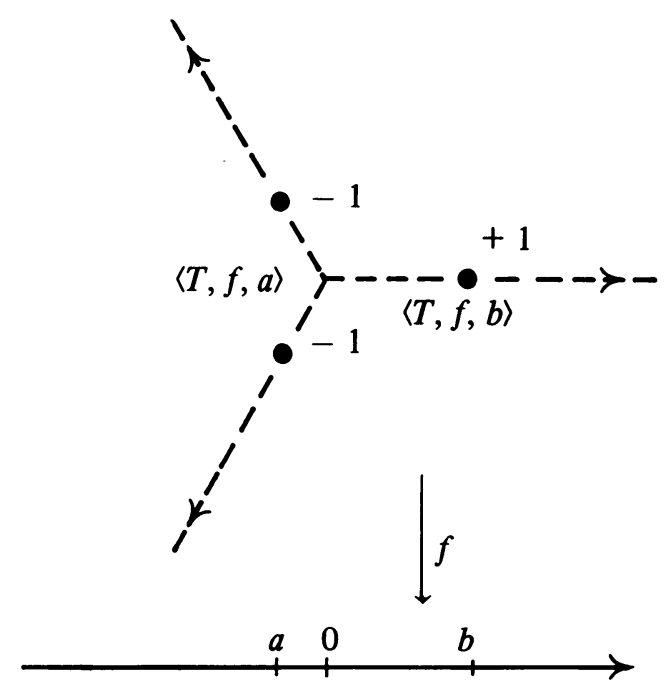

Figure 2 
Nevertheless, letting $S$ be the congruence class modulo 3 of $T$, we infer that $\mathrm{spt}^{3} \partial S=\varnothing$, that $\langle S, f, 0\rangle^{3}$ is the congruence class of the two congruent chains $-2 \delta_{(0,0)}, \delta_{(0,0)}$ and that the slice modulo $3,\langle S, f, \cdot\rangle^{3}$, is continuous on all of $\mathbf{R}$, in accord with the slicing modulo $\nu$ theorem.

It follows from this theorem and the remarks in 4.4 that all of the results of $[6,4.3-5.11]$ carry over to the modulo $\nu$ case, including the resulting intersection theory.

The notation employed in the present paper is consistent with [6] and [4] (see the glossaries of [4, pp. 669-671]). As in [6] we let, for any two maps $f$ : $A \rightarrow B$ and $g: A \rightarrow C$, the mapping $f \bullet g: A \rightarrow B \times C$ be defined by $(f \square g)(a)=$ $(f(a), g(a))$ for $a \in A$.

Chains modulo $\nu$ arose classically in algebraic topology as members of various chain complexes having coefficient group $\mathrm{Z}_{\nu}=\mathrm{Z} / \nu \mathrm{Z}$ [8, III, 4 (c)]. Rectifiable and flat chains modulo $\nu$ (especially $\nu=2$ ) have been used in geometric measure theory in the study of multidimensional elliptic variation problems ([9], [5], [1], [2], [4, 5.3.21]). In [7] analytic chains and analytic chains modulo $\nu$ are used to define homology theories with coefficient groups $\mathbf{Z}$ and $\mathbf{Z}_{\nu}$ for the category of pairs of semianalytic sets and continuous maps between such pairs; questions concerning the extent of local orientability of real analytic varieties are treated there in these terms.

2. Locally flat chains modulo $\nu$. The basic reference for flat chains modulo $\nu$ in $\mathbf{R}^{n}$ is $[4,4.2 .26]$. In this section we assume $M$ is a separable Riemannian manifold and state appropriate generalizations of some of the results of $[6, \S 3]$ and $[4,4.2 .26]$ for chains in $M$ whose supports are not necessarily compact.

Recalling $[6, \$ 3]$ and $\left[4\right.$, p. 423] we endow the group $\mathfrak{F}_{k}^{\text {loc }}(M)$ of $k$ dimensional locally integral flat chains in $M$ with the modulo $\nu$ topology by associating with each pair $(U, \delta)$ such that

$$
U \text { is open, Clos } U \text { is compact, } \delta>0 \text {, }
$$

a basic neighborhood of $0, \mathbf{N}^{\nu}(U, \delta)$, consisting of those chains $T \in \mathcal{F}_{k}^{\operatorname{loc}}(M)$ for which there exist $Q \in R_{k}(M), R \in R_{k}(M)$, and $S \in R_{k+1}(M)$ with spt $(T-R-\partial S-\nu Q)$ $C M \sim U, \mathbf{M}(R)+\mathbf{M}(S)<\delta$.

The statements and proofs of $[6,3,3.1,3.2(1)(3)(4)(5), 3.3,3.4]$ readily generalize to the modulo $\nu$ case. Assuming $\left\{U_{1}, U_{2}, \cdots\right\}$ is a cover of $M$ consisting of open sets with compact closures, we also see that the modulo $y$ topology is induced by the complete pseudometric dist $^{\nu}$ defined by

where

$$
\operatorname{dist}^{\nu}\left(T_{1}, T_{2}\right)=\sum_{j=1}^{\infty}\left[\delta_{j} / 2^{j}\left(1+\delta_{j}\right)\right]
$$




$$
\delta_{j}=\inf \left\{\delta: T_{1}-T_{2} \in \mathbf{N}^{\nu}\left(U_{j}, \delta\right)\right\}
$$

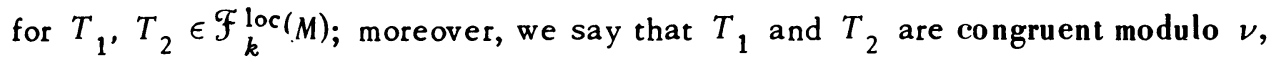
and write $T_{1} \equiv T_{2} \bmod \nu$, whenever $\operatorname{dist}^{\nu}\left(T_{1}, T_{2}\right)=0$. The resulting congruence classes, which we call $k$ dimensional locally flat chains modulo $\nu$ in $M$, are the cosets of the factor group

$$
\mathcal{F}_{k}^{\text {loc, } \nu}(M)=\mathscr{F}_{k}^{\text {loc }}(M) / \mathcal{F}_{k}^{\text {loc }}(M) \cap\left\{T: \operatorname{dist}^{\nu}(T, 0)=0\right\}
$$

this group is complete in the induced metric. The operations on $\mathcal{F}_{k}$ loc $(M)$ of restriction $[6,3.3]$, boundary (for $k \geq 1$ ), and $f_{\#}$ (see $[6,3.0(3)]$ ) induce similar continuous operations on $\mathcal{F}_{k}^{\text {loc }, \nu}(M)$. For $T \in \mathcal{F}_{k}^{\text {loc }}(M)$ we let $(T)^{\nu} \in \mathcal{F}_{k}^{\text {loc }, \nu}(M)$ be the coset of $T$. We also define the modulo $\nu$ supports:

$$
\begin{array}{ll}
\operatorname{spt}^{\nu} S=\bigcap_{R \in S} \operatorname{spt} R & \text { for } S \in \mathcal{F}_{k}^{\text {loc, } \nu}(M), \\
\operatorname{spt}^{\nu} T=\operatorname{spt}^{\nu}(T)^{\nu} & \text { for } T \in \mathcal{F}_{k}^{\text {loc }}(M) .
\end{array}
$$

From the discussion of $[4, \mathrm{p} .430]$ we infer that two locally rectifiable currents $T_{1}, T_{2} \in R_{k}^{\operatorname{loc}}(M)$ are congruent modulo $\nu$ if and only if there exists a third chain $Q \in \mathfrak{R}_{k}^{l \propto c}(M)$ such that $T_{1}-T_{2}=\nu Q$; if, moreover, $T_{1}$ and $T_{2}$ are analytic chains in $M[6,4]$, then $Q$ is also an analytic chain because

$$
\text { spt } Q \subset \text { spt } T_{1} \cup \text { spt } T_{2}, \quad \text { spt } \partial Q \subset \text { spt } \partial T_{1} \cup \text { spt } \partial T_{2} \text { • }
$$

3. A constancy lemma and some consequences.

3.1. Lemma (compare $[4,4.1 .31(2)])$. Suppose $B$ is a connected proper $k$ dimensional class 1 submanifold of $M$. If $Q, R \in \mathcal{F}_{k}^{l o c}(M),\left(\operatorname{spt}^{\nu} Q\right) \sim B$ and $\left(\operatorname{spt}^{\nu} R\right) \sim B$ are closed, and $\left(\mathrm{spt}^{\nu} \partial Q\right) \cup\left(\mathrm{spt}^{\nu} \partial R\right) \subset M \sim B$, then there exist integers $i$ and $j$ in $\{0,1, \cdots, \nu-1\}$ such that

$$
\begin{gathered}
\Theta^{k}(\|Q\|, x) \equiv i \bmod \nu \quad \text { and } \Theta^{k}(\|R\|, x) \equiv j \bmod \nu \text { for all } x \in B, \\
\operatorname{spt}^{\nu}(j Q-i R) \subset M \sim B .
\end{gathered}
$$

Proof. For any $x \in B$, there exist neighborhoods $U \subset$ Clos $U \subset V$ of $x$ and a class 1 map $p$ from $V$ into $\mathbf{R}^{k}$ such that $V \cap \operatorname{spt}^{\nu} Q \subset B, V \cap \operatorname{spt}^{\nu} R \subset B$, $p \mid B \cap V$ is a proper class 1 diffeomorphism and $C=p(U \cap B)$ is an open cube in $\mathbf{R}^{k}$. Observing that $p_{\#}(Q \mid V)\left\llcorner C\right.$ and $p_{\#}(R \mid V)\llcorner C$ extend $[6,3.3]$ to flat chains $Q^{\prime}$ and $R^{\prime}$ in $R^{k}$ with

$$
\begin{aligned}
\operatorname{spt}^{\nu} \partial Q^{\prime} & \subset\left[\operatorname{spt}^{\nu} \partial p_{\#}(Q \mid V)\right] \cup \operatorname{spt}\left(\partial \left[p_{\#}(Q \mid V)\llcorner C]-\left[\partial p_{\#}(Q \mid V)\llcorner C]\right)\right.\right. \\
& \subset p\left[V \cap \operatorname{spt}^{\nu} \partial Q\right] \cup \operatorname{Bdry} C=\text { Bdry } C, \\
\text { spt }^{\nu} \partial R^{\prime} & \subset \text { Bdry } C,
\end{aligned}
$$


we infer from the modulo $\nu$ version of the second proposition of $[4,4.2 .3]$ that

$$
Q^{\prime} \equiv i \mathbf{E}^{k}\left\llcorner C \bmod \nu \text { and } R^{\prime} \equiv j \mathbf{E}^{k}\llcorner C \bmod \nu\right.
$$

for some $i$ and $j$ in $\{0,1, \cdots, \nu-1\}$; hence,

$$
\begin{aligned}
& \Theta^{k}(\|Q\|, x) \equiv i \quad \bmod \nu \text { and } \Theta^{k}(\|R\|, x) \equiv j \bmod \nu \text { for all } x \in U \cap B, \\
& U \cap \operatorname{spt}^{\nu}(j Q-i R) \subset p^{-1}\left[C \cap \operatorname{spt}^{\nu}\left(j Q^{\prime}-i R^{\prime}\right)\right]=\varnothing
\end{aligned}
$$

by the modulo $\nu$ analogue of the second corollary of $[4,4.1 .15]$.

If follows that the sets

$$
\begin{aligned}
B_{i, j}= & {\left[B \sim \operatorname{spt}^{\nu}(j Q-i R)\right] } \\
& \cap\left\{x: \Theta^{k}(\|Q\|, x) \equiv i \bmod \nu \text { and } \Theta^{k}(\|R\|, x) \equiv j \bmod \nu\right\}
\end{aligned}
$$

for $i$ and $j$ in $\{0,1, \cdots, \nu-1\}$ form a relatively open disjointed cover of $B$; thus $B_{i, j}=B$ for some $i, j$.

3.2. Proposition (compare $[3,3.17],[6,3.6])$. Suppose $f$ is a locally Lipschizian map of $M$ into an open subset $N$ of $\mathbf{R}^{n}, T \in \mathbb{R}_{k}^{l o c}(M)$, and $\mu$ is a positive integer such that $f \mid \mathrm{spt}^{\nu} T$ is proper, $\left(\mathrm{spt}^{\nu} T\right) \sim \mathrm{spt}^{\nu} \partial T$ is locally connected, and $N \cap\left\{y: \operatorname{card}\left(f^{-1}\{y\} \cap \mathrm{spt}^{\nu} T\right) \leq \mu\right\}$ is dense in $N \sim f\left(\mathrm{spt}^{\nu} \partial T\right)$. Let

$$
Y=\left[N \sim f\left(\operatorname{spt}^{\nu} \partial T\right)\right] \cap\left\{y: \operatorname{card}\left(f^{-1}\{y\} \cap \operatorname{spt}^{\nu} T\right)<\infty\right\},
$$

$G$ be the class of all nonempty connected open subsets of $N \sim f\left(\operatorname{spt}^{\nu} \partial T\right)$ and, for $W \in G, \Gamma(W)$ be the set of all components of $f^{-1}(W) \cap$ spt $^{\nu} T$; also let

$$
H=\bigcup\{\Gamma(W): W \in G\}, \quad H^{*}=\left\{V \cap f^{-1}(Y): V \in H\right\} .
$$

Then the following eight conclusions bold:

(1) For each $V \in \Gamma(W)$ there exists an integer $\Delta(V) \in\{0,1, \cdots, \nu-1\}$ such that $f_{\#}(T<V) \equiv \Delta(V) \mathbf{E}^{n}\llcorner W \bmod \nu$.

(2) If $V \in \Gamma(W)$ and $\Delta(V) \neq 0$, then $f(V)=W$.

(3) $\operatorname{Card}[\Gamma(W) \cap\{V: \Delta(V) \neq 0\}] \leq \mu$.

(4) If $W \subset W^{\prime}$ belong to $G$ and $V^{\prime} \in \Gamma\left(W^{\prime}\right)$, then $\Delta\left(V^{\prime}\right) \equiv \Sigma_{V^{\prime} \supset V \in \Gamma(W)} \Delta(V)$ $\bmod \nu$.

(5) The family $H^{*}$ is a base for the relative topology of $f^{-1}(Y) \cap \mathrm{spt}^{\nu} T$.

(6) If $x \in f^{-1}(Y) \cap \mathrm{spt}^{\nu} T$, then $\Delta(V)$ bas the same value, hereafter denoted $\Delta(x)$, for all sufficiently small neighborboods $V$ of $x$ belonging to $H$.

(7) If $y \in Y$, then

$$
f_{\#}\left[T<f^{-1}(W)\right] \equiv\left[\sum_{x \in f^{-1}\{y\} \cap \mathrm{spt}} \nu_{T} \Delta(x)\right] \mathrm{E}^{n}\llcorner W \bmod \nu
$$


for all sufficiently small neighborboods $W$ of $y$ belonging to $G$.

(8) The function mapping $y \in Y$ onto $\Sigma_{x \in f}-1\{y\} \cap_{\mathrm{spt}} \nu_{T} \Delta(x) \delta_{x}$ is mass bounded by $\mu \nu$ and continuous in the modulo $\nu$ topology.

Proof. To prove (1) we observe that

$$
\begin{aligned}
& W \cap \operatorname{spt}^{\nu} \partial f_{\#}\left(T \llcorner V ) \subset f \left[f^{-1}(W) \cap \operatorname{spt}^{\nu} \partial(T\llcorner V)]\right.\right. \\
& \quad \subset f\left(\left[f^{-1}(W) \cap \operatorname{spt}^{\nu} \partial T\right] \cup\left[f^{-1}(W) \cap \operatorname{spt}^{\nu} T \cap \text { Bdry } V\right]\right)=\varnothing,
\end{aligned}
$$

and apply 3.1 with $B=W, Q=\mathbf{E}^{n} \mathrm{~L} W$, hence $i=1$, and $R=f_{\#}(T\llcorner V)$ to choose $\Delta(V) \in\{0,1, \cdots, \nu-1\}$ such that

$$
\begin{aligned}
& \operatorname{spt}^{\nu}\left[f_{\#}(T<V)-\Delta(V) \mathbf{E}^{n}\llcorner W] \subset \mathbf{R}^{n} \sim W,\right. \\
& f_{\#}\left(T\llcorner V)=f_{\#}\left(T \llcorner V ) \left\llcornerW \equiv \Delta(V) \mathbf{E}^{n}\llcorner W \bmod \nu .\right.\right.\right.
\end{aligned}
$$

We verify (2) by noting that if $\Delta(V) \neq 0$, then

$$
\begin{gathered}
W \subset W \cap \operatorname{spt}^{\nu} f_{\#}(T L V) \subset f\left[f^{-1}(W) \cap \operatorname{spt}^{\nu}(T L V)\right] \\
\subset f\left[f^{-1}(W) \cap \operatorname{spt}^{\nu} T \cap C l o s V\right]=f(V) .
\end{gathered}
$$

To prove (3) we choose a point $y \in W$ with card $\left(f^{-1}\{y\} \cap \operatorname{spt}^{\nu} T\right) \leq \mu$ and apply (2).

For the proof of (4) we have only to modify the proof of $[3,3.7$ (4)] by changing the first and last equalities to congruences modulo $\nu$.

(5) and (6) follow just as in the proofs of $[3,3.17$ (5) (6)].

To prove (7) we abbreviate $F=f^{-1}\{y\} \cap \operatorname{spt}^{\nu} T$, choose open sets $W_{x}$ and disjoint sets $V_{x}$ for $x \in F$ so that $y \in W_{x} \in G, x \in V_{x} \in \Gamma\left(W_{x}\right)$, and $f_{\#}\left(T L V_{x}\right)$ $\equiv \Delta(x) \mathbf{E}^{n} L W_{x} \bmod \nu$, and observe that $F$ is finite,

$$
\delta=\operatorname{dist}\left[y,\left(\bigcup_{x \in F} \operatorname{Bdry} W_{x}\right) \cup f\left(\operatorname{spt}^{\nu} T \sim \bigcup_{x \in F} V_{x}\right)\right]
$$

is positive, and any $W \in G$ contained in $\mathbf{U}(y, \delta)$ satisfies

$$
\begin{aligned}
f^{-1}(W) \cap \operatorname{spt}^{\nu} T & \subset \bigcup_{x \in F} V_{x} \text { and } \\
f_{H}\left[T\left\llcorner f^{-1}(W)\right]\right. & \equiv \sum_{x \in F} f_{H}\left(T L\left[V_{x} \cap f^{-1}(W)\right]\right) \\
& \equiv \sum_{x \in F} \Delta(x) \mathbf{E}^{n}\left\llcorner\left(W_{x} \cap W\right)=\left(\sum_{x \in F} \Delta(x)\right) \mathbf{E}^{n}\llcorner W \bmod \nu .\right.
\end{aligned}
$$

The mass bound in (8) follows from (6) and (3). A proof of the modulo $\nu$ continuity results from modifying the wording of the proof of $[6,3.6(9)]$ by changing spt $T$ to spt $^{\nu} T$ and the second equality in the computation of $\partial S$ to a congruence modulo $\nu$. 
4. Analytic chains modulo $\nu$. Assuming now that $M$ is a separable $m$ dimensional real analytic Riemannian manifold, we first recall the notion of real analytic dimension (which is defined in $[6,2.2]$ ):

If $A$ is an analytic subset of $M$, then $\operatorname{dim} A=\sup \{-1, k: A$ contains a nonempty $k$ dimensional analytic submanifold of $M$ \}.

If $E$ is an arbitrary subset of $M$, then $\operatorname{dim} E=\inf \left\{k: E \subset \bigcup_{U \in \mathcal{U}} A_{U}\right.$ for some locally-finite open cover $\mathcal{U}$ of $M$ and $k$ dimensional analytic subsets $A_{U}$ of $U$ for $U \in \mathcal{U}\}$.

We will call $S$ a $k$ dimensional analytic chain modulo $\nu$ in $M$ if and only if (compare [6, §4]) $S \in \mathcal{F}_{k}^{l o c, \nu}(M), \operatorname{dim}\left(\operatorname{spt}^{\nu} S\right) \leq k, \operatorname{dim}\left(\operatorname{spt}^{\nu} \partial S\right) \leq k-1$. It then follows that there exists a $k$ dimensional analytic chain $T$ belonging to $S$ with spt $T=\operatorname{spt}^{\nu} S$.

In fact, for every $x \in M$ there exist, by [4, 3.4.8 (11)], an open ball $U$ about $x$ and a finite family $B$ of disjoint $k$ dimensional orientable analytic blocks $B$ $[6, \S 2]$ in $U \cap\left(\mathrm{spt}^{\nu} S \sim \mathrm{spt}^{\nu} \partial S\right)$ with orienting $k$ vectorfields $\beta_{B}$ such that the real analytic dimension of $Z=U \cap\left(\operatorname{spt}^{\nu} S \sim \operatorname{spt}^{\nu} \partial S \sim \bigcup B\right)$ is less than $k$. Applying, for each $B \in \mathfrak{B}, 3.1$ to $Q=\left(\mathcal{H}^{k}\llcorner B) \wedge \beta_{B}\right.$ and any locally flat chain $R$ belonging to $S$ (hence, $i=1$, $\operatorname{spt}^{\nu} R=\mathrm{spt}^{\nu} S$, and $\mathrm{spt}^{\nu} \partial R=\mathrm{spt}^{\nu} \partial S$ ), we choose an integer $j_{B} \in\{1,2, \cdots, \nu-1\}$ so that

$$
\operatorname{spt}^{\nu}\left[S-\left(j_{B}\left[\mathcal{H}^{k} L B\right] \wedge \beta_{B}\right)^{\nu}\right]=\operatorname{spt}^{\nu}\left[R-j_{B}\left(\mathcal{H}(k L B) \wedge \beta_{B}\right] \subset M \sim B .\right.
$$

Then

$$
U \cap \operatorname{spt}^{\nu} S-\left[\left(\sum_{B \in B} j_{B}\left[\mathcal{H}^{k} L B\right] \wedge \beta_{B}\right)^{\nu}\right]
$$

is contained in $Z \cup \operatorname{spt}^{\nu} \partial S$, has $\mathcal{H}^{k}$ measure zero by $[6,2.2$ (5) (4)], and is empty by $\left[4,4.2 .26(4.2 .14)^{2}\right]$. Moreover

$$
U \cap \mathrm{spt}^{\nu} S=U \cap \operatorname{Clos} \bigcup \mathfrak{B}=U \cap \mathrm{spt} \sum_{B \in \mathbb{B}} j_{B}\left(\mathcal{H}^{k} L B\right) \wedge \beta_{B} .
$$

To construct $T$ globally we choose open balls $U_{1}, U_{2}, \ldots$ along with analytic chains $T_{1}, T_{2}, \cdots$ in $M$ such that $\left\{U_{1}, U_{2}, \cdots\right\}$ is a locally finite open cover of $M$ and

$$
U_{i} \cap \operatorname{spt}^{\nu}\left[S-\left(T_{i}\right)^{\nu}\right]=\varnothing, \quad U_{i} \cap \mathrm{spt}^{\nu} S=U_{i} \cap \operatorname{spt} T_{i}
$$

Then we use $[6,2.2(7)]$ to select, for $i \in\{1,2, \cdots\}$, open balls $V_{i}$ with closure in $U_{i}$ such that $M \subset \bigcup_{i=1}^{\infty} V_{i}$ and

$$
\mathcal{H}^{k}\left(\left[\left(\operatorname{spt}^{\nu} S\right) \cup\left(\operatorname{spt} T_{i}\right)\right] \cap \operatorname{Bdry} V_{j}\right)=0
$$


for $i$ in $\{0,1, \cdots\}$ and $j$ in $\{0,1, \cdots, \nu-1\}$, define the a nalyt ic chain

$$
T=T_{1}\left\llcorner V_{1}+\sum_{i=2}^{\infty} T_{i}\left\llcorner\left(V_{i} \sim \bigcup_{j=1}^{i-1} V_{j}\right)\right. \text {, }\right.
$$

and conclude that spt $T=\operatorname{spt}^{\nu} T$ and that $\mathcal{H}^{k}\left(\operatorname{spt}^{\nu}\left[S-(T)^{\nu}\right]\right)=0$; hence $S=$ $(T)^{\nu}$ by $\left[4,4.2 .26(4.2 .14)^{\nu}\right]$.

4.1. Proof of the Slicing Modulo $\nu$ Theorem. We fix an analytic chain $T$ belonging to $S$ with spt $T=\operatorname{spt}^{\nu} S$; let

$W=\mathbf{R}^{n} \cap\left\{w: \operatorname{dim}\left(f^{-1}\{w\} \cap \operatorname{spt} T\right) \leq k-n\right.$ and $\operatorname{dim}\left(f^{-1}\{w\} \cap\right.$ spt $\left.\left.\partial T\right) \leq k-n-1\right\}$,

hence $W \subset Y$, and observe that it suffices to prove the following statement:

There exists a continuous map $\mathcal{S}$ from $Y$ into the $k-n$ dimensional analytic chains modulo $\nu$ in $M$ such that $\mathcal{S}(w)=(\langle T, f, w\rangle)^{\nu}$ for every $w \in W$.

In fact suppose this statement is true and $T^{\prime}$ is another analytic chain belonging to $S$ with

$$
\begin{aligned}
& W^{\prime}=\mathbf{R}^{n} \cap\left\{w: \operatorname{dim}\left(f^{-1}\{w\} \cap \operatorname{spt} T^{\prime}\right) \leq k-n\right. \text { and } \\
& \left.\qquad \operatorname{dim}\left(f^{-1}\{w\} \cap \operatorname{spt} \partial T^{\prime}\right) \leq k-n-1\right\} .
\end{aligned}
$$

Then $T^{\prime}-T=\nu Q$ for some $k$ dimensional analytic chain $Q$, and for any $w \epsilon$ $W^{\prime} \cap W$,

$$
\operatorname{dim}\left(f^{-1}\{w\} \cap \operatorname{spt} Q\right) \leq k-n, \quad \operatorname{dim}\left(f^{-1}\{w\} \cap \operatorname{spt} \partial Q\right) \leq k-n-1 ;
$$

hence, by $[6,4.3],\langle Q, f, w\rangle$ is an analytic chain in $M$, and

$$
\left\langle T^{\prime}, f, w\right\rangle-\langle T, f, w\rangle=\nu\langle Q, f, w\rangle \equiv 0 \bmod \nu .
$$

The two continuous maps $\mathcal{S} \mid W^{\prime}$ and $\left(\left\langle T^{\prime}, f, \cdot\right\rangle\right)^{\nu}$ of $W^{\prime}$ agree on the dense [6, $2.2(7)]$ subset $W^{\prime} \cap \cap W$, hence are equal.

To prove the statement we consider three cases.

Case 1. $M$ is an open subset of $\mathbf{R}^{m}$, spt $T$ is compact, and $k=n$. Here we use 3.1 and [4, 3.4.8(11)], reasoning as in [4, 4.2.28] to see that $\operatorname{spt}^{\nu} T \sim$ $\mathrm{spt}^{\nu} \partial T$ is locally connected, note that $f \mid$ spt $T$ is proper because spt $T$ is compact, choose, according to $[6,2.11$ (1)] a positive integer $\mu$ so that the set

$$
\mathbf{R}^{n} \cap\left\{y: \operatorname{card}\left(f^{-1}\{y\} \cap \operatorname{spt} T\right)>\mu\right\}
$$

has Lebesgue measure zero, and then apply 3.2 (7) by setting

$$
\mathcal{S}(y)=\left(\sum_{x \in f^{-1}\{y\} \cap \mathrm{spt}^{\nu} T} \Delta(x) \delta_{x}\right)^{\nu} \text { for } y \in Y .
$$


The statement follows by comparing 3.2 (8) (6) (1) and [6, 3.6 (8) (6) (1)].

Case 2. $M$ is an open subset of $\mathbf{R}^{m}$, spt $T$ is compact, and $k>n$. Here we assume, for contradiction, that the statement is false. Since $W$ is dense in $\mathbf{R}^{n}$ by $[6,2.2(7)]$, there then must exist a point $y \in Y$ such that $(\langle T, f, w\rangle)^{\nu}$ fails to converge as $w$ approaches $y$ in $W$. Assured by [4, 4.2.17] and $[6,4.2]$ that the family $\{\langle T, f, w\rangle: w \in W\}$ is relatively compact in $\mathbf{I}_{k}(M)$ we choose two sequences $w_{1,1}, w_{1,2}, w_{1,3} \cdots$ and $w_{2,1}, w_{2,2}, w_{2,3} \cdots$ in $W$ converging to $y$ and integral currents $L_{1}$ and $L_{2}$ in $\mathbf{I}_{k}(M)$ such that $\operatorname{spt}^{\nu}\left(L_{1}-L_{2}\right)$ is nonempty and $\left\langle T, f, w_{i, j}\right\rangle$ approaches $L_{i}$, for $i \in\{1,2\}$, as $j$ approaches $\infty$. Since

$$
\mathrm{spt}^{\nu} L_{i} \subset f^{-1}\{y\} \cap \mathrm{spt}^{\nu} S, \quad \mathrm{spt}^{\nu} \partial L_{i} \subset f^{-1}\{y\} \cap \mathrm{spt}^{\nu} \partial S,
$$

$\left(L_{i}\right)^{\nu}$ is an analytic chain modulo $\nu$ in $M$ for $i \in\{1,2\}$. Thus we may select an open set $U$ in $M \sim \operatorname{spt}^{\nu} \partial\left(L_{1}-L_{2}\right)$ and an orthogonal projection $p: M \rightarrow \mathbf{R}^{k-n}$ such that $B=U \cap \operatorname{spt}^{\nu}\left(L_{1}-L_{2}\right)$ is a nonempty connected $k-n$ dimensional analytic submanifold of $M$ and $p \mid B$ is an analyt ic isomorphism. Fixing, by [6, $2.2(7)], z \in p(B)$ so that

$$
\operatorname{dim}\left(f^{-1}\{y\} \cap p^{-1}\{z\} \cap \operatorname{spt}^{\nu} S\right)=0, \quad f^{-1}\{y\} \cap p^{-1}\{z\} \cap \operatorname{spt}^{\nu} \partial S=\varnothing
$$

we infer from $3.1,3.2(6)$, and Case 1 that

$$
\left\langle\left(L_{1}-L_{2}\right)^{\nu}, p, z\right\rangle^{\nu} \mid U \neq(0)^{\nu} .
$$

Next using $[6,3.2]$ we pass to subsequences, without changing notations, so that there exist for $i \in\{1,2\}$ and $j \in\{1,2, \cdots\}$ rectifiable chains $R_{i, j} \epsilon$ $R_{k-n}(M), S_{i, j} \in R_{k-n+1}(M)$ with

$$
\begin{aligned}
& \operatorname{spt}\left(L_{i}-\left\langle T, f, w_{i, j}\right\rangle-R_{i, j}-\partial S_{i, j}\right) \subset M \sim U, \\
& \mathbf{M}\left(R_{i, j}\right)+\mathbf{M}\left(S_{i, j}\right) \leq j^{-1 \varrho^{k-n} \mathbf{U}\left(z, j^{-1}\right),}
\end{aligned}
$$

and then apply $[6,2.2(7)]$ and $[4,4.3 .6,4.3 .2(2)]$ to choose points $z_{i, j} \in \mathbf{R}^{k-n}$ $\cap \mathbf{U}\left(z, j^{-1}\right)$ such that

$$
\begin{aligned}
& \operatorname{dim}\left(p^{-1}\left\{z_{i, j}\right\} \cap\left[\left(f^{-1}\left\{w_{i, j}\right\} \cap \text { spt } T\right) \cup \operatorname{spt} L_{i}\right]\right) \leq 0, . \\
& p^{-1}\left\{z_{i, j}\right\} \cap\left[\left(f^{-1}\left\{w_{i, j}\right\} \cap \operatorname{spt} \partial T\right) \cup \operatorname{spt} \partial L_{i}\right]=\varnothing, \\
& \left\langle R_{i, j}, p, z_{i, j}\right\rangle \in \mathbb{R}_{0}(M), \quad\left\langle S_{i, j}, p, z_{i, j}\right\rangle \in R_{1}(M), \\
& \mathbf{M}\left\langle R_{i, j}, p, z_{i, j}\right\rangle+\mathbf{M}\left\langle s_{i, j}, p, z_{i, j}\right\rangle \leq j^{-1},
\end{aligned}
$$

and deduce from $[6,3.5(2)]$ that the slices

$$
\left\langle L_{i}-\left\langle T, f, w_{i, j}\right\rangle, p, z_{i, j}\right\rangle\left|U=\left[\left\langle R_{i, j}, p, z_{i, j}\right\rangle+(-1)^{k-n} \partial\left\langle S_{i, j}, p, z_{i, j}\right\rangle\right]\right| U
$$


approach 0 as $j$ approaches $\infty$ for $i \in\{1,2\}$. Finally applying Case 1 twice and $[6,4.5]$, we obtain the desired contradiction by computing

$$
\begin{aligned}
(0)^{\nu} & \neq\left(\left(L_{1}-L_{2}\right)^{\nu}, p, z\right\rangle^{\nu}\left|U=\lim _{j \rightarrow \infty}\left(\left\langle L_{1}, p, z_{1, j}\right\rangle-\left\langle L_{2}, p, z_{2, j}\right\rangle\right)^{\nu}\right| U \\
& =\lim _{j \rightarrow \infty}\left(\left\langle\left\langle T, f, w_{1, j}\right\rangle, p, z_{1, j}\right\rangle-\left\langle\left\langle T, f,\left(w_{2, j}\right\rangle, p, z_{2, j}\right\rangle\right)^{\nu} \mid U\right. \\
& =\lim _{j \rightarrow \infty}\left(\left\langle T, f \boxminus p,\left(w_{1, j}, z_{1, j}\right)\right\rangle-\left\langle T, f \boxminus p,\left(w_{2, j}, z_{2, j}\right)\right\rangle\right)^{\nu} \mid U \\
& =\left(\left\langle(T)^{\nu}, f \boxminus p,(y, z)\right\rangle^{\nu}-\left\langle(T)^{\nu}, f \boxminus p,(y, z)\right\rangle^{\nu}\right) \mid U .
\end{aligned}
$$

Case 3. General case. Assuming the theorem false, we choose, by the modulo $\nu$ analogue of $[6,3.2(1)]$ an analytic isomorphism $\phi$ of some open subset $V$ of $M$ onto $\mathbf{R}^{m} \cap \mathbf{U}(0,2), U=\phi^{-1}[\mathbf{U}(0,1)]$, a countable subset $C$ of $W$, and a point $y \in Y \cap \operatorname{Clos} C$ such that $\langle T, f, w\rangle^{\nu} \mid U=\langle T|U, f| U, w\rangle^{\nu}$ fails to converge as $w$ approaches $y$ in $C$. Choosing, by [6, $2.2(7)], r$ between 1 and 2 such that

$$
\begin{aligned}
& \operatorname{dim}\left(|\phi|^{-1}\{r\} \cap \text { spt } T\right) \leq k-1, \\
& \operatorname{dim}\left(|\phi|^{-1}\{r\} \cap f^{-1}\{y\} \cap \text { spt } T\right) \leq k-n-1, \\
& \operatorname{dim}\left(|\phi|^{-1}\{r\} \cap f^{-1}\{w\} \cap \text { spt } T\right) \leq k-n-1,
\end{aligned}
$$

we infer that

$$
\left\langle\phi_{\#}(T \mid V)\left\llcorner\mathbf{U}(0, r), f \circ \phi^{-1}, w\right\rangle^{\nu}\right| \mathbf{U}(0,1)=(\phi \mid U)_{\#}\langle T|U, f| U, w\rangle^{\nu}
$$

fails to converge as $w$ approaches $y$ in $C$, which contradicts either Case 1 or Case 2 with $S, T, f$ replaced by $\left[\phi_{\#}(T \mid V)\llcorner\mathbf{U}(0, r)]^{\nu}, \phi_{\#}(T \mid V)\llcorner\mathbf{U}(0, r), f \circ\right.$ $\phi^{-1}$.

4.2. Remark. With $T, f, Y, W$ as in 4.1 , we see that the equation $(\langle T, f, y\rangle)^{\nu}$ $=\left\langle(T)^{\nu}, f, y\right\rangle^{\nu}$ holds whenever $y \in W$. For $y \in Y \sim W$ however, this may fail to be true even though the slice $\langle T, f, y\rangle$, as defined in $[4,4.3]$, is an analytic chain in $M$. For example if $f: \mathbf{R} \rightarrow \mathbf{R}$ is the identity map and

$$
T=\mathbf{E}^{1}\left\llcorner\{x: x>0\}-\mathbf{E}^{1}\llcorner\{x: x<0\},\right.
$$

then

$$
(\langle T, f, 0\rangle)^{2}=(0)^{2} \neq\left(\delta_{0}\right)^{2}=\left\langle(T)^{2}, f, 0\right\rangle^{2}
$$

4.3. For $S, f, Y$ as in the Slicing Modulo $\nu$ Theorem and $y \in Y$, we readily infer, by continuity and the definition of the slice $[4,4.3 .1]$, the following four statements: 
(1) $\operatorname{spt}^{\nu}\langle S, f, y\rangle^{\nu} \subset f^{-1}\{y\} \cap \operatorname{spt}^{\nu} S$.

(2) $\partial\langle S, f, y\rangle^{\nu}=(-1)^{n}\langle\partial S, f, y\rangle^{\nu}$ in case $k>n$.

(3) $\langle S, f, y\rangle^{\nu} \mid U=\langle S|U, f| U, y\rangle^{\nu}$ whenever $U$ is an open subset of $M$.

(4) $\langle S, b \circ f, b(y)\rangle^{\nu}=\langle S, f, y\rangle^{\nu}$ whenver $b$ is an orientation-preserving analytic isomorphism of $\mathbf{R}^{n}$.

4.4. We will now discuss how all of the statements and most of the proofs of $[6,4.4-5.11]$ carry over to the modulo $\nu$ case. The wording of these propositions should be modified by replacing "chain, spt, $\mathcal{F}_{*}^{\operatorname{loc}}(M)$, and $\langle, \quad\rangle$ " to "chain modulo $\nu, \operatorname{spt}^{\nu}, \mathcal{F}_{*}^{l o c}, \nu$, and $\langle,,\rangle^{\nu, "}$. We will number such generalizations by using the superscript $\nu$ above the number of the corresponding proposition of [6].

$(4.4)^{\nu}$ Here it is necessary to define $\left\langle f_{\#} T, g, y\right\rangle^{\nu}$ as $f_{\#}\left(\langle T, g \circ f, y\rangle^{\nu}\right)$ and then observe by 4.2 and $[6,4.4]$ that this is consistent in case $f_{\#} T$ is an analytic chain modulo $\nu$.

$(4.5)^{\nu}$ requires a new proof; we prove the first conclusion by considering two cases:

Case 1. $s=n+l$. Here, assuming the theorem false, we choose a point

$$
x \in \operatorname{spt}^{\nu}\left[\langle S, f \boxminus g,(a, b)\rangle^{\nu}-\left\langle\langle S, f, a\rangle^{\nu}, g, b\right\rangle^{\nu}\right],
$$

then an open ball $U$ about $x$ in $M$ and $i$ and $j$ in $\{0,1, \cdots, \nu-1\}$ so that

$$
\begin{gathered}
U \cap \mathrm{spt}^{\nu} \partial S=\varnothing, \quad(\operatorname{Clos} U) \cap f^{-1}\{a\} \cap g^{-1}\{b\} \cap \mathrm{spt}^{\nu} S=\{x\}, \\
\langle S, f \boxminus g,(a, b)\rangle^{\nu}\left|U=i\left(\delta_{x}\right)^{\nu}\right| U \neq j\left(\delta_{x}\right)^{\nu}\left|U=\left\langle\langle S, f, a\rangle^{\nu}, g, b\right\rangle^{\nu}\right| U .
\end{gathered}
$$

Since

$$
\begin{aligned}
0<\delta & =\inf \left\{|(a, b)-(f \boxminus g)(w)|: w \in(\operatorname{Bdry} U) \cap \mathrm{spt}^{\nu} S\right\} \\
& \leq \inf \left\{|b-g(w)|: w \in(\operatorname{Bdry} U) \cap \operatorname{spt}^{\nu}\langle S, f, a\rangle^{\nu}\right\},
\end{aligned}
$$

$V=U \cap(f \boxminus g)^{-1}(U[(a, b), \delta])$ is open and the two functions, $(f \mid V) \square(g \mid V) \mid \operatorname{spt}^{2} S$ and $(g \mid V) \mid \mathrm{spt}^{\nu}\langle S, f, a)^{\nu}$ are proper maps. Applying $3.2(7)$ twice-with $M, N, f$, and $T$ replaced:

first, by $V, \mathbf{U}[(a, b), \delta],(f \mid V) \bullet(g \mid V)$, and any a nalytic chain belonging to $S \mid V$, and

second, by $V, \mathbf{U}(b, \delta), g \mid V$, and any analytic chain belonging to $\langle S, f, a\rangle^{\nu} \mid V$, we choose open balls, $W$ about $a$ in $\mathbf{R}^{n}$ and $Z$ about $b$ in $\mathbf{R}^{l}$, and

$$
\begin{aligned}
& a=f\left|U \cap(f \boxminus g)^{-1}(W \times Z), \quad \beta=g\right| U \cap(f \boxminus g)^{-1}(W \times Z), \\
& R=S \mid U \cap(f \boxminus g)^{-1}(W \times Z)
\end{aligned}
$$

such that $W \times Z \subset \mathbf{U}[(a, b), \delta]$ and

$$
(\alpha \boxminus \beta)_{\sharp} R=i\left(\mathbf{E}^{n} \times \mathbf{E}^{l}\right)^{\nu}\left|(W \times Z), \quad \beta_{\sharp}\langle R, a, a\rangle^{\nu}=j\left(\mathbf{E}^{l}\right)^{\nu}\right| Z .
$$


Letting $q: W \times Z \rightarrow W, b: U \cap(f \bullet g)^{-1}(W \times Z) \rightarrow W \times Z$ be given by $q(w, z)=$ $w, b(u)=(a, \beta(u))$ for $(w, z) \in W \times Z$ and $u \in U \cap(f \square g)^{-1}(W \times Z)$, we use (4.4) $)^{\nu}$ and the modulo $\nu$ version of $[4,4.1 .15]$ to compute

$$
\begin{aligned}
i\left(\delta_{a} \times \mathbf{E}^{l}\right)^{\nu} \mid(W \times Z) & =\left\langle(\alpha \boxminus \beta)_{\#} R, q, a\right\rangle^{\nu}=(\alpha \boxminus \beta)_{\#}\langle R, a, a\rangle^{\nu}=b_{\#}\langle R, a, a\rangle^{\nu} \\
& =\left[\left(\delta_{a}\right)^{\nu} \mid W\right] \times \beta_{\#}\langle R, a, a\rangle^{\nu}=j\left(\boldsymbol{\delta}_{a} \times \mathbf{E}^{l}\right)^{\nu} \mid(W \times Z),
\end{aligned}
$$

hence $i=j$, a contradiction.

Case 2. $s>n+l$. Again assuming the theorem is false, we choose an open set $U$ in $M \sim \operatorname{spt}^{\nu} \partial S$ and an analytic map $p: U \rightarrow \mathbf{R}^{s-n-l}$ such that

$$
B=U \cap \operatorname{spt}^{\nu}\left(\langle S, f \boxminus g,(a, b)\rangle^{\nu}-\left\langle\langle S, f, a\rangle^{\nu}, g, b\right\rangle^{\nu}\right)
$$

is a nonempty connected analyt ic submanifold of $M$ and $p \mid B$ is an analytic isomorphism, let $w \in p(B)$, abbreviate $\bar{S}=S|U, \bar{f}=f| U, \bar{g}=g \mid U$ and use 3.1, 3.2 (6) (1), Case 1, and 4.3 (4) to derive the contradiction

$$
\begin{aligned}
(0)^{\nu} & \neq\left\langle\langle\bar{S}, \bar{f} \boxminus \bar{g},(a, b)\rangle^{\nu}-\left\langle\langle\bar{S}, \bar{f}, a\rangle^{\nu}, \bar{g}, b\right\rangle, p, w\right\rangle^{\nu} \\
& =\left\langle\langle\bar{S}, \bar{f} \boxminus \bar{g},(a, b)\rangle^{\nu}, p, w\right\rangle^{\nu}-\left\langle\langle\bar{S}, \bar{f}, a\rangle^{\nu}, \bar{g} \boxminus p,(b, w)\right\rangle^{\nu} \\
& =\langle\bar{S},(\bar{f} \boxminus \bar{g}) \boxminus p,((a, b), w)\rangle^{\nu}-\langle\bar{S}, \bar{f} \boxminus(\bar{g} \boxminus p),(a,(b, w))\rangle^{\nu}=(0)^{\nu} .
\end{aligned}
$$

The second conclusion of $(4.5)^{\nu}$ is readily obtained from the first as in $[6$, 4.5].

$(4.6)^{\nu}$ through $(4.9)^{\nu}$ now follows as in $[6,4.6-4.9]$.

In $(\$ 5)^{\nu}, M$ and $N$ need only be orientable modulo $\nu$ with orienting modulo $\nu$ cycles $\pi, \pi$. The intersection modulo $\nu$ of $Q$ and $R, Q \cap^{\nu} R \in \mathcal{F}_{q+r-m}^{\text {boc, }}(M)$, is to be defined in a manner similar to $[6,5.0$ (1)] where $b$ now need only preserve orientation modulo $\nu$; also we make the obvious generalization to obtain the notion that $\{S, T\}$ intersect suitably modulo $\nu$.

For $(5.1)^{\nu}$ we assume now that $Q$ and $R$ are analytic chains modulo $\nu$ satisfying

$$
\begin{array}{ll}
\operatorname{dim}\left(b^{-1}\{y\} \cap \mathrm{spt}^{\nu} Q\right) \leq q-k, & \operatorname{dim}\left(b^{-1}\{y\} \cap \mathrm{spt}^{\nu} \partial Q\right) \leq q-k-1, \\
\operatorname{dim}\left(c^{-1}\{z\} \cap \mathrm{spt}^{\nu} R\right) \leq r-l, & \operatorname{dim}\left(c^{-1}\{z\} \cap \mathrm{spt}^{\nu} \partial R\right) \leq r-l-1,
\end{array}
$$

and obtain the desired equations by continuity from $[6,5.1]$.

$(5.2)^{\nu}$ through $(5.6)^{\nu}$ may now be deduced as in $[6,5.2-5.6]$.

In $(5.7)^{\nu}, L$ is an analytic chain modulo $\nu, \pi=\left(\mathbf{E}^{m} \mid M\right)^{\nu}$, and $\eta=\left(\mathbf{E}^{n} \mid N\right)^{\nu}$, and we apply $[6,5.7]$ to any member $T$ of $L$ to infer that 


$$
\begin{aligned}
\tau_{\#}\langle M \times L, f \circ \sigma, 0\rangle^{\nu} & =\left(\tau_{\#}\left\langle\left(\mathbf{E}^{m} \mid M\right) \times T, f \circ \sigma, 0\right\rangle\right)^{\nu}=(T)^{\nu}=L \\
& =(-1)^{(l+n) n}\left(\tilde{\sigma}_{\#}\left\langle T \times\left(\mathbf{E}^{n} \mid N\right), \tilde{f} \circ \tilde{\tau}, 0\right\rangle\right)^{\nu} \\
& =(-1)^{(l+n) n} \tilde{\sigma}_{\#}\langle L \times \pi, \tilde{f} \circ \tilde{\tau}, 0\rangle^{\nu} .
\end{aligned}
$$

$(5.8)^{\nu}$ through $(5.11)^{\nu}$, Case 4, follow from the proofs of $[6,5.8-5.10$, Case $4]$ by use of 3.2 in place of $[4,4.1 .31]$ and $\left[4,4.2 .28(4.2 .14)^{\nu}\right]$ in place of $[4,4.1$. 20].

To modify the proof of Case 5 it suffices to use 3.2 (7) to choose $\rho$ and $\sigma$ small enough so that

$$
\begin{aligned}
(f \mid V)_{H}[(S \times T) \mid V] & =(-1)^{(m-s) t_{i}}\left[\mathbf{E}^{m} \mid \mathbf{U}(0, \sigma)\right]^{\nu} . \\
\langle(S \times T)|V, f| V, 0\rangle^{\nu} & =(-1)^{(m-s) t_{i}}\left(\delta_{x} \mid V\right)^{\nu} .
\end{aligned}
$$

For Case 6 , instead of using $[6,4.1]$, we observe that

If $R$ is a $k$ dimensional analytic cbain modulo $\nu$ in an open subset $M$ of $\mathbf{R}^{m}$ and $\left\langle R, \mathbf{p}_{\lambda} \mid M, y\right\rangle^{\nu}$ is zero for all $\lambda \in \Lambda(m, k)$ and $\varrho^{k}$ almost all $y$ in $\mathbf{R}^{k}$, then $R$ equals zero.

In fact, otherwise for any regular point $x$ of $\operatorname{spt}^{\nu} R \sim \mathrm{spt}^{\nu} \partial R$ there is a $\lambda \in \Lambda(m, k)$ such that $\operatorname{dim} \mathbf{p}_{\lambda}\left[\operatorname{Tan}\left(\operatorname{spt}^{\nu} R, x\right)\right]=k$, and, by $[4,3.1 .18]$, an open neighborhood $U$ of $x$ in $M \sim \mathrm{spt}^{\nu} \partial R$ such that $B=U \cap \mathrm{spt}^{\nu} R$ is a connected analytic submanifold of $M$ and $p_{\lambda} \mid B$ is an analytic isomorphism. Then by 3.1, $\left(\mathbf{p}_{\lambda} \mid U\right)_{\#}(R \mid U)^{\nu}$ equals $\left[j \mathbf{E}^{k} \mid \mathbf{p}_{\lambda}(B)\right]^{\nu}$ for some $j \in\{1,2, \cdots, \nu-1\}$; hence, by 3.2 ,

$$
\left\langle R, \mathbf{p}_{\boldsymbol{\lambda}} \mid M, \mathbf{p}_{\boldsymbol{\lambda}}(x)\right\rangle^{\nu} \mid U=\left\langle R\left|U, \mathbf{p}_{\boldsymbol{\lambda}}\right| U, \mathbf{p}_{\boldsymbol{\lambda}}(x)\right\rangle^{\nu}=\left(j \boldsymbol{\delta}_{\boldsymbol{x}} \mid U\right)^{\nu} \neq(0)^{\nu}
$$

whenver $x \in B$, a contradiction.

Finally Case 7 follows from Case 5, Case 6, and $(5.11)^{\nu}$ (3) (4) as before.

\section{BIBLIOGRAPHY}

1. F. J. Almgren, Jr., Some interior regularity theorems for minimal surfaces and an extension of Bernstein's theorem, Ann. of Math. (2) 84 (1966), 277-292. MR 34 \#702.

2. - Existence and regularity almost everwhere of solutions to elliptic variational problems among surfaces of varying topological type and singularity structure, Ann. of Math. (2) 87 (1968), 321-391. MR 37 \#837.

3. H. Federer, Some theorems on integral currents, Trans. Amer. Math. Soc. 117 (1965), 43-67. MR 29 \#5984.

4. - - Geometric measure theory, Die Grundlehren der math. Wissenschaften, Band 153, Springer-Verlag, New York, 1969. MR 41 \#1976.

5. W. H. Fleming, Flat chains over a finite coefficient group, Trans. Amer. Math. Soc. 121 (1966), 160-186. MR 32 \#2554.

6. R. M. Hardt, Slicing and intersection theory for chains associated with real analytic varieties, Acta. Math. 129 (1972), 75-136.

7. - Homology of real analytic and semianalytic sets, Ann. Scuola Norm. Sup. Pisa (to appear). 
8. S. Lefschetz, Algebraic topology, Amer. Math. Soc. Colloq. Publ., vol. 27, Amer. Math. Soc., Providence, R. I., 1942. MR 4, 84.

9. W. P. Ziemer, Integral currents mod 2, Trans. Amer. Math. Soc. 105 (1962), 496524. MR 27 \#268.

DEPARTMENT OF MATHEMATICS, BROWN UNIVERSITY, PROVIDENCE, RHODE ISLAND 02912

DEPARTMENT OF MATHEMATICS, UNIVERSITY OF MINNESOTA, MINNEAPOLIS, MINNESOTA 55455 